\title{
Self-Oscillating Structural Polymer Gels
}

\author{
Srinivasa R. Pullela ${ }^{1}$, Qingsheng Wang ${ }^{2,3}$, Zhengdong Cheng ${ }^{1,4^{*}}$ \\ ${ }^{1}$ Artie McFerrin Department of Chemical Engineering, Texas A\&M University, College Station, USA \\ ${ }^{2}$ Department of Fire Protection \& Safety, Oklahoma State University, Stillwater, USA \\ ${ }^{3}$ School of Chemical Engineering, Oklahoma State University, Stillwater, USA \\ ${ }^{4}$ Materials Science and Engineering Program, Texas A\&M University, College Station, USA \\ Email: ${ }^{2}$ zcheng@mail.che.tamu.edu, qingsheng.wang@okstate.edu
}

Received November 2, 2012; revised January 22, 2013; accepted January 29, 2013

Copyright (C) 2013 Srinivasa R. Pullela et al. This is an open access article distributed under the Creative Commons Attribution License, which permits unrestricted use, distribution, and reproduction in any medium, provided the original work is properly cited.

\begin{abstract}
Self-oscillating polymer gel has become a distinguished class of smart soft materials. Here we fabricated and demonstrated a self-oscillating structural gel network with the incorporation of the Belousov-Zhabotinsky (BZ) reaction. The structural polymer gel oscillates at a macroscopic level with remarkably faster kinetics compared to a normal gel of similar chemical compositions. The structural polymer gel also displays larger oscillating amplitude compared to the normal gel because of the increased diffusion of fluids surrounding the gel particles. This type of structural polymer gels can be harnessed to provide novel and feasible applications in a wide variety of fields, such as drug delivery, nanopatterning, chemical and biosensing, and photonic crystals.
\end{abstract}

Keywords: Structural Gel; Belousov-Zhabotinsky Reaction; Self-Oscillation; Poly-( $N$-isopropylacrylamide); Fast Response

\section{Introduction}

The Belousov-Zhabotinsky (BZ) reaction, which is a well-known nonlinear dynamic chemical system, has attracted much attention due to their fascinating phenomena $[1,2]$. The reaction includes a series of metalion-catalyzed oxidative reactions of organic substrates, such as malonic acid, by an acidified bromate solution. Recently, the BZ reaction has been used to induce mechanical oscillations in polymeric systems to mimic biological materials $[3,4]$. In particular, Yoshida and his co-workers have developed a self-oscillating cross-linked gel composed of stimuli-responsive poly-( $N$-isopropylacrylamide) (PNIPAM) polymer with grafted Ru(bipy) ${ }_{3}$ moiety as the $\mathrm{BZ}$ catalyst $[5,6]$. The $\mathrm{BZ}$ reaction generates autonomous and rhythmical redox oscillations from the oxidized $\mathrm{Ru}(\mathrm{III})$ state to the reduced $\mathrm{Ru}(\mathrm{II})$ state, which induced a periodic volume oscillation in the PNIPAM gel when the gels are immersed in an aqueous acidic solution containing the substrates for the BZ reaction [7]. Due to the periodic mechanical oscillations, a novel biomimetic self-walking gel was developed by Yoshida et al. [8]. Directional movement of gel is produced by asymmetrical swelling-deswelling of the PNIPAM-co-Ru(bipy) ${ }_{3}$ gels.

\footnotetext{
${ }^{*}$ Corresponding author.
}

An approach was also developed recently for simulateing chemo-responsive gels that exhibit not only large variations in volume but also alterations in shape [9]. Through this approach, oscillating gels undergoing the $\mathrm{BZ}$ reaction were simulated, which showed that the formation of the wave pattern depends on the aspect ratios of the sample. Such self-oscillating polymer gel has become a distinguished class of soft materials which are anticipated for new concept applications such as self-beating pacemakers and drug delivery systems synchronized with human circadian rhythms [10-12]. Such advanced materials, especially those stimuli-responsive polymers and gels have been actively investigated due to a unique nature of volume change in response to external stimuli [13]. These self-oscillating gel particles have various potential industrial applications, such as drug delivery [14], chemical and biosensing [15], fabrication of photonic crystals [16] and absorbents [17].

In our previous studies, we have prepared the PNIPAM gel particles of uniform size using a two step synthesis with covalently bound $\mathrm{Ru}(\text { bipy })_{3}$ catalyst modifying the process used by Yoshida and his co-workers $[18,19]$. The volume responses of PNIPAM particles in coupling to the $\mathrm{BZ}$ reaction as a function of 
substrate concentrations and temperature have been characterized [19]. It has shown that the automatic mechanical oscillation of the gel particles was induced by the covalently coupled BZ reaction. The chemical oscillations of the $\mathrm{BZ}$ reaction induce the periodic charge oscillation of the Ruthenium ions, which leads to the periodic change of the hydrophilicity of the PNIPAM polymer network, hence the volume oscillation of the microgel particles. In this swell/shrink process, water is diffused either from the surface towards the centre of the gel, or vice versa.

However, the slow response and small amplitude of the responsive PNIPAM polymer gels prevent their further usages [20], because the swell/shrink rate is inversely proportional to the square of the distance that the water molecules have to travel [21]. Therefore, the gels with a fast response and large amplitude are desired for feasible applications. If we can improve the frequency and amplitude of the self-oscillation, the autonomous gel systems will have a much broader range of applications. One idea to solve this problem is to raise the temperature in the system so that the $\mathrm{BZ}$ reaction rate increases. However, the conventional-type self-oscillating gel usually shrinks at temperatures above its LCST (lower critical solution temperature) [4]. Recently, Cho et al. fabricated a fast responsive structural gel scaffold, where microgel particles assembled either through bridging or depletion interactions to yield a structure that response to the external stimulus at a macroscopic level in much shorter times as compared to a bulk polymer gel of similar characteristics [22]. In this work, we further develop this technique to prepare a self-oscillating structural PNIPAM gel that contains a catalyst for the BZ reaction to achieve an autonomous oscillation with both high frequency and large amplitude, without the temperature limitation.

\section{Experimental Section}

\subsection{Synthesis of PNIPAM-co-Ru(bipy) ${ }_{3}$ Gels}

Surfactant-free PNIPAM microgels have been prepared by a precipitation polymerization of $\mathrm{N}$-isopropylacrylamide (NIPAM). In a typical procedure, NIPAM (4 g), allylamine $(250 \mu \mathrm{L}), \mathrm{Ru}(\mathrm{vmbipy})(\text { bipy })_{2} \mathrm{PF}_{6}(0.200 \mathrm{~g}), \mathrm{N}$, N-methylenebisacrylamide (0.180 g), 2,2'-azobisisobutyronitrile (AIBN) $(0.166 \mathrm{~g})$ and water $(80 \mathrm{~mL})$ were added in a $200 \mathrm{ml}$ round-bottomed flask reactor equipped with a stirrer, a condenser, a nitrogen inlet, and a thermometer. The contents were heated to $70^{\circ} \mathrm{C}$ under a nitrogen atmosphere and at a stir rate of $300 \mathrm{rpm}$. The polymerization was continued for 6 hours. The microgel dispersion was then filtered through a filter $(\sim 100 \mu \mathrm{m}$ mesh size) to remove some aggregates generated during polymerization and then, cooled down to room temperature.

\subsection{Synthesis of Clusters of PNIPAM-co-Ru(bipy) ${ }_{3}$ Gels}

Clusters of microgels were made by linking microgel particles covalently using glutaraldehyde. To the microgel suspension prepared above, $0.002 \mathrm{M}$ negatively charged polyacrylic acid solution (Mol. Wt. 400,000) was added to induce ionic attractions with the positively charged Ruthenium bipyridine and allylamine. The dispersion was heated to $60^{\circ} \mathrm{C}$ leading to a phase separation of the microgel from the continuous aqueous phase. Then, approximately 1 volume \% of glutaraldehyde aqueous solution $(50 \% \mathrm{w} / \mathrm{v}$, Aldrich) was added to chemically link amide groups on the surface of the microgel particles in contact with each other. After a 1 hour reaction at $60^{\circ} \mathrm{C}$, the resulting gel scaffolds were washed with DI water. This cluster of microgel particles with interconnected PNIPAM gels was named as "structural gel" and its self-oscillating behavior was compared with that of "normal gel" with no interconnected gel network. The "normal gel" was prepared following the preparation method by Maeda et al. [23]. For compared studies, both structural gel and normal gel were cut into the same dimensions $(5 \times 1 \times 1 \mathrm{~mm})$ and the swell/shrink response was measured at both oxidized and reduced states of Ruthenium ion. The synthetic scheme of the structural gel using PNIPAM particles is shown in Scheme 1.

\subsection{Synthesis of Suspensions of PNIPAM-co-Ru(bipy) ${ }_{3}$ Gel Particles}

The suspensions of normal gel particles and structural gel particles were prepared as below. The gel particles of PNIPAM with $\mathrm{Ru}$ (bipy) ${ }_{3}$ were synthesized by emulsion polymerization as follows: purified NIPAM (3.80 g), $\mathrm{Ru}(\text { bipy })_{3}(0.422 \mathrm{~g})$, allylamine $(0.200 \mathrm{~g})$, sodium dodecylbenzene sulfonate $(0.700 \mathrm{~g}), \mathrm{N}, \mathrm{N}$-methylenebisacrylamide $(0.700 \mathrm{~g})$, and AIBN $(0.169 \mathrm{~g})$ were added in $200 \mathrm{~mL}$ of $\mathrm{H}_{2} \mathrm{O}$. The suspension was stirred at $60^{\circ} \mathrm{C}$ for 8 hours under the $\mathrm{N}_{2}$-flow condition. The resulting mixture was purified through dialysis against pure water for 14 days. To the $100 \mathrm{~mL}$ of the above suspension, $0.002 \mathrm{M}$ of polyacrylamide solution and 5 volume $\%$ of glutaraldehyde were added at room temperature. The mixture was stirred at $250 \mathrm{rpm}$ for 48 hours under $\mathrm{N}_{2}$-flow condition to form the suspension of structural gel particles.

\section{Results and Discussion}

For the self-oscillation study, the structural gels were dispersed in aqueous solution containing the reactants of the $\mathrm{BZ}$ reaction at a fixed concentration: Malonic acid (MA) $0.30 \mathrm{M}$, sodium bromate $\left(\mathrm{NaBrO}_{3}\right) 0.75 \mathrm{M}$, and nitric acid $\left(\mathrm{HNO}_{3}\right) 1.0 \mathrm{M}$. It was observed that in the oxidized state of the metal catalyst Ruthenium, the equilibrium volume of the gel was larger than that in the reduced 


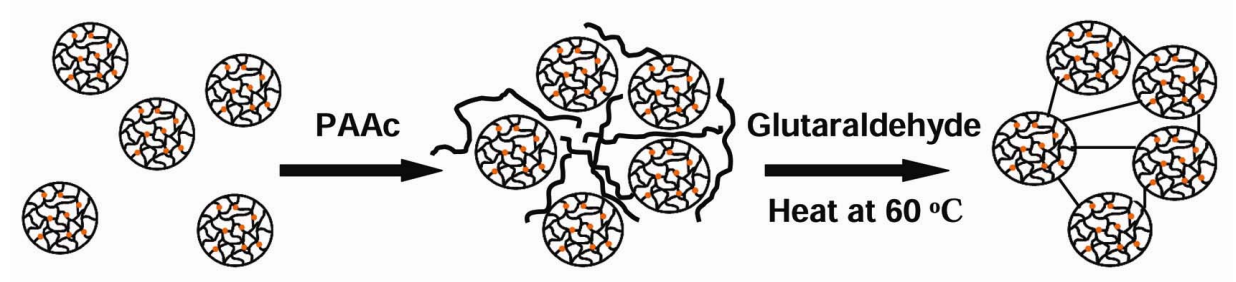

Scheme 1. Preparation of PNIPAM-co-Ru(bipy) $)_{3}$ structural gel particles. It only shows the frame structure, not detailed molecules.

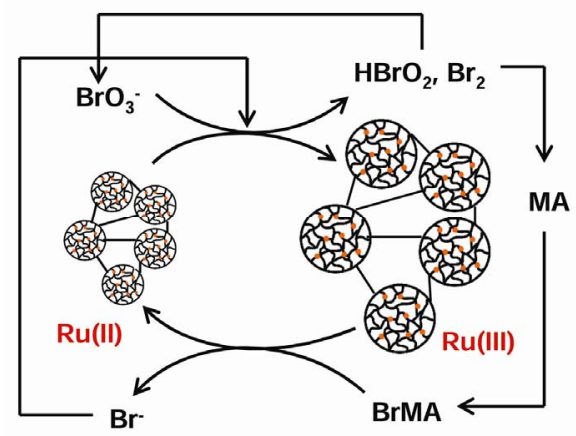

Figure 1. Mechanical self-oscillation of PNIPAM-co-Ru(bipy) ${ }_{3}$ structural gel particles.

state in all temperature conditions. The automatic mechanical oscillation of the PNIPAM-co-Ru(bipy) ${ }_{3}$ structural gels, induced by the BZ reaction, is shown in Figure 1.

Initially, the metal catalyst $\mathrm{Ru}(\mathrm{II})$ is in the reduced state, and the structural gels remain in the shrink state. With the introduction of BZ substrates, after some time, and during the rest of the induction time, $\mathrm{Ru}(\mathrm{II})$ switches to its oxidized form, $\mathrm{Ru}(\mathrm{III})$. In this state, the structural gels become swollen. This oscillation behavior is caused by the significantly different solubility of the $\mathrm{Ru}$ (bipy) ${ }_{3}$ moiety in its oxidized and reduced states. The reduced $\mathrm{Ru}$ (bipy) ${ }_{3}$ moiety in the gel has an extreme hydrophobic property while the oxidized $\mathrm{Ru}(\text { bipy })_{3}$ part in the gel has a great hydrophilic property [4].

For the suspension of PNIPAM structural gel particles, under constant temperature and stirring conditions together with BZ substrates, the time course of transmission was monitored by use of UV-vis spectroscopy. The transmittance of the mixed solutions in the $190-856 \mathrm{~nm}$ wavelength range was measured continuously in an episodic data capture mode. The transmittance is correlated with the volume change (i.e., gel particles shrink when transmittances decrease). The oscillations in transmittance at $460 \mathrm{~nm}$ and $685 \mathrm{~nm}$ reflect the chemical oscillation of the $\mathrm{Ru}$ (bipy) $)_{3}$ catalyst between the $\mathrm{Ru}$ (II) and $\mathrm{Ru}(\mathrm{III})$ states, whereas the oscillations at $570 \mathrm{~nm}$ are attributable to the conformation change of the PNIPAM polymers [19]. Figure 2 shows the normalized transmittance of the structural gel as a function of temperature which is measured under different oxidization states of the Ruthenium. Compared to the suspension of normal gel particles, the suspension of structural gel particles displays much higher swelling and shrinkage. The interconnected structured gel particles shrink to $\sim 40 \%$ of their original size above their LCST (lower critical solution temperature) whereas the normal gel particles shrink to $\sim 55 \%$. It should be noted that the particle size of the suspension is $160 \mathrm{~nm}$ for the normal gel particles and about $700 \mathrm{~nm}$ for the structural gel particles. Since several normal gel particles are clustered together in the latter, oscillations are expected to be higher in structural gel suspensions.

The response of the prepared structural gel is compared with that of the normal gel at room temperature and shown in Figure 3. The structural gel exhibits a remarkable improvement in response dynamics. As mentioned above, both structural and normal gels were cut into the dimensions of $5 \times 1 \times 1 \mathrm{~mm}$. The shrinkage of gels in length with time was monitored by placing in a solution of $1.0 \mathrm{M}$ nitric acid containing either $\mathrm{Ce}(\mathrm{IV})$ or $\mathrm{Ce}(\mathrm{III})$, which corresponds to the oxidized or reduced states of the Ruthenium. The degree of swelling in the oxidized state is higher than the swelling in the reduced state for both gels due to the increased Donnan osmotic pressure. It is observed that the structural gel responds 10 times faster than the normal gel. This remarkably faster kinetics may arise from the smaller dimensions of the gel particles than form the three dimensional gel networks.

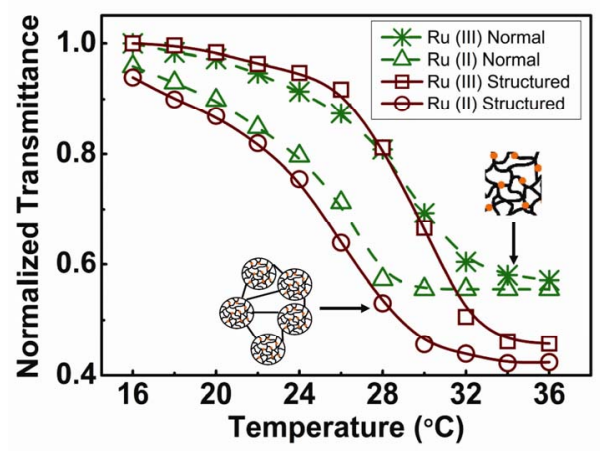

Figure 2. Large volume oscillations of PNIPAM-co-Ru(bipy) structural gels in comparison to normal gels as a function of temperature. 


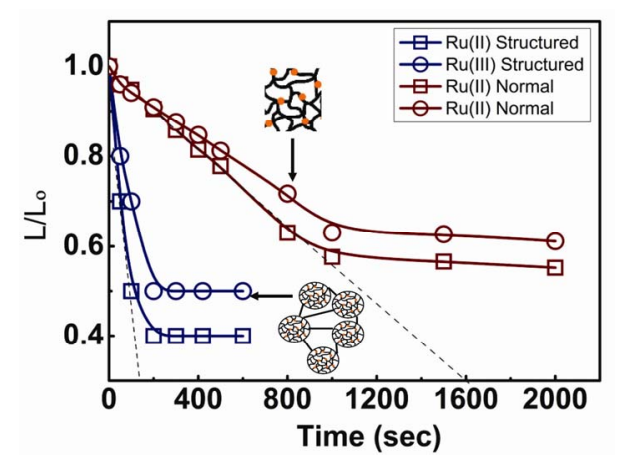

Figure 3. Response time of PNIPAM-co-Ru(bipy) $)_{3}$ structural gels in both oxidized and reduced states of the Ruthenium metal in comparison with the response time of normal gels.
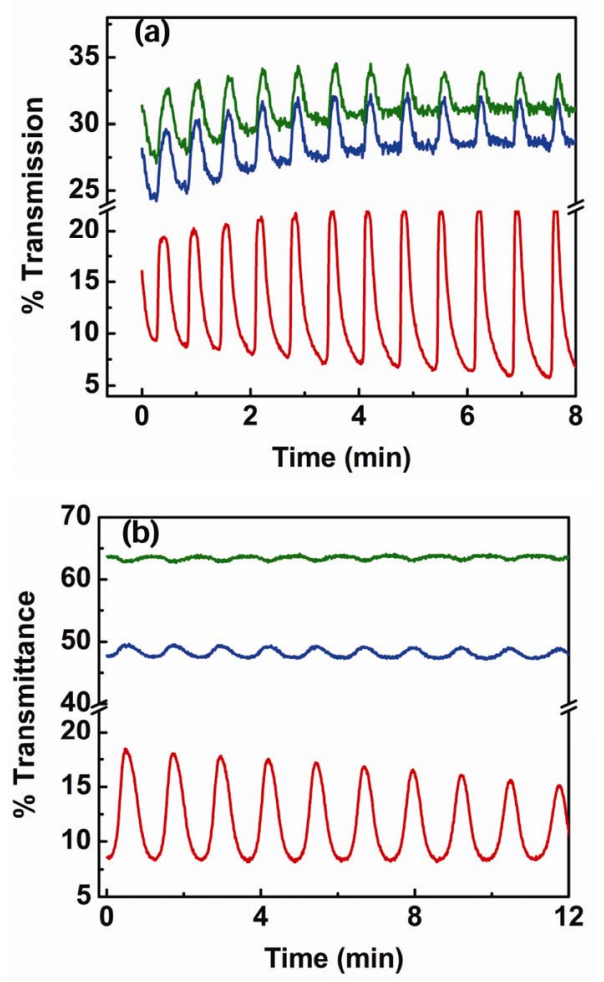

Figure 4. Comparison of the oscillation frequency and amplitude between self-oscillating BZ active gels: (a) structural gels and (b) normal gels. Chemical oscillations were observed at $460 \mathrm{~nm}$ and $685 \mathrm{~nm}$ as shown in the green and blue lines, and mechanical oscillations were observed at 570 $\mathrm{nm}$ as shown in the red line.

The smaller size speeds up the diffusion of the fluid through the gel networks. In the case of normal gels, the cross-linked network reduces the diffusion of surrounding fluids and hence the gel exhibits slow response.

The oscillation frequency and amplitude changes of the PNIPAM gel particles in the structural gel and normal gel suspension are compared under the same BZ reaction conditions as mentioned above and shown in
Figure 4. The $\mathrm{Ru}(\text { bipy })_{3}$ complex has different absorption spectra in the reduced $\mathrm{Ru}(\mathrm{II})$ state and the oxidized $\mathrm{Ru}(\mathrm{III})$ state as an inherent property. The upper two oscillations (at wavelengths of $460 \mathrm{~nm}$ and $685 \mathrm{~nm}$ ) reflect the chemical oscillation of the $\mathrm{Ru}(\text { bipy })_{3}$ catalyst between the $\mathrm{Ru}(\mathrm{II})$ and $\mathrm{Ru}(\mathrm{III})$ states, whereas the bottom oscillations (at the wavelength $570 \mathrm{~nm}$ ) are attributable to the conformation change of the PNIPAM polymers [19]. It should be noted that the upper two oscillations in Figures 4(a) and (b) are mostly out of phase. However, the shape of the oscillation for structural gel particles is most pulse-like (non-sinusoidal) compared to normal gel particles. As Figure 4 shows, particles in the structural gel oscillate at a rate twice faster than that of the gel particles prepared by normal polymerization method. The oscillation amplitude of particles in the structural gel is also much larger than that of normal gel particles.

Based on these special properties obtained from the experiments (fast response kinetics with large amplitudes), more possible applications by the use of these fast responsive and large amplitude polymer gels could be identified, such as reduced detection time of chemical and bio sensors, micro-fluidic valves that operate with faster open/close mechanisms, mechanical switches, and expansion or contraction of bio-related mechanisms such as rapid artificial muscle movements, faster heart beat in the case of artificial heart in which expansions or contractions will occur quickly to drive a mechanism. However, much more refined systematic work should be performed to explain the phenomena in this complex system in the future. First, the different mechanical oscillations may be related to the space distribution of the catalyst even at the macroscopic level and the presence of other moieties in structural polymer gels (i.e., some amine moieties). Second, the strongly confined chemical systems such as the gel network in this work may significantly affect the effective kinetics. Last but not least, the gel suspensions are similar to segregated reactive fluids where micro-mixing problems may apply, which suggest that the chemical dynamics could be affected by differences in the segregated fluids [24].

\section{Conclusion}

In summary, we have succeeded in fabricating a selfoscillating structural polymer gel coupling to the $\mathrm{BZ}$ reaction, which exhibits fast response kinetics with large amplitudes. There are two keys to our approach: one is the incorporation of the $\mathrm{BZ}$ chemistry into responsive polymer gels and thus to power a mechanical action; another one is the use of microgel particles that can be assembled through bridging interactions to yield a structure that oscillates at a macroscopic level. Particles in the structural gel oscillate twice faster than that of the gel particles prepared by normal polymerization method. The approach we have presented in this study offers a new 
way of fabricating controllable and functional selfoscillating polymer gels with excellent responsive capabilities in a wide range.

\section{Acknowledgements}

This work was partially funded by the National Science Foundation (DMR-1006870). The authors wish to thank Texas A\&M University and Oklahoma State University for the research support.

\section{REFERENCES}

[1] I. R. Epstein, "Nonlinear Oscillations in Chemical and Biological Systems," Physica D, Vol. 51, No. 1-3, 1991, pp. 152-160. doi:10.1016/0167-2789(91)90228-2

[2] R. J. Field, E. Koros and R. Noyes, "Oscillations in Chemical Systems. II. Thorough Analysis of Temporal Oscillation in the Bromate-Cerium-Malonic Acid System," Journal of the American Chemical Society, Vol. 94, No. 25, 1972, pp. 8649-8664. doi:10.1021/ja00780a001

[3] R. Yoshida, T. Sakai, O. Tabata and T. Yamaguchi, "Design of Novel Biomimetic Polymer Gels with Self-Oscillating Function," Science and Technology of Advanced Materials, Vol. 3, No. 2, 2002, pp. 95-102. doi:10.1016/S1468-6996(02)00010-4

[4] S. Nakamaru, S. Maeda, Y. Hara and S. Hashimoto, "Control of Autonomous Swelling-Deswelling Behavior for a Polymer Gel," Journal of Physical Chemistry B, Vol. 113, No. 14, 2009, pp. 4609-4613. doi:10.1021/jp811228y

[5] R. Yoshida, T. Takahashi, T. Yamaguchi and H. Ichijo, "Self-Oscillating Gel," Journal of the American Chemical Society, Vol. 118, No. 21, 1996, pp. 5134-5135. doi:10.1021/ja9602511

[6] Y. Hara and R. Yoshida, "Control of Oscillating Behavior for the Self-Oscillating Polymer with pH-Control Site," Langmuir, Vol. 21, No. 21, 2005, pp. 9773-9776. doi:10.1021/la052070v

[7] Y. Hara and R. Yoshida, "Self-Oscillation of Polymer Chains Induced by the Belousov-Zhabotinsky Reaction under Acid-Free Conditions," Journal of Physical Chemistry B, Vol. 109, No. 19, 2005, pp. 9451-9454. doi:10.1021/jp0501704

[8] S. Maeda, Y. Hara, T. Sakai, R. Yoshida and S. Hashimoto, "Self-Walking Gel," Advanced Materials, Vol. 19, No. 21, 2007, pp. 3480-3484. doi:10.1002/adma.200700625

[9] V. V. Yashin and A. C. Balazs, "Pattern Formation and Shape Changes in Self-Oscillating Polymer Gels," Science, Vol. 314, No. 5800, 2006, pp. 798-801. doi:10.1126/science.1132412

[10] V. V. Yashin, O. Kuksenok and A. C. Balazs, "Modeling Autonomously Oscillating Chemo-Responsive Gels," Progress in Polymer Science, Vol. 35, No. 1-2, 2010, pp. 155-173. doi:10.1016/j.progpolymsci.2009.10.003

[11] R. Yoshida, "Self-Oscillating Gels Driven by the Belousov-Zhabotinsky Reaction as Novel Smart Materials," Advanced Materials, Vol. 22, No. 2010, pp. 3463-3483. doi:10.1002/adma.200904075

[12] R. Yoshida, "Self-oscillating Polymer Gel as Novel Biomimetic Materials Exhibiting Spatiotemporal Structure," Colloid and Polymer Science, Vol. 289, No. 5-6, 2011, pp. 475-487. doi:10.1007/s00396-010-2371-y

[13] T. Asoh, M. Matsusaki, T. Kaneko and M. Akashi, "Fabrication of Temperature-Responsive Bending Hydrogels with a Nanostructured Gradient," Advanced Materials, Vol. 20, No. 11, 2008, pp. 2080-2083. doi:10.1002/adma.200702727

[14] C. E. Reese, A. V. Mikhonin, M. Kamenjicki, A. Tikhonov and S. A. Asher, "Nanogel Nanosecond Photonic Crystal Optical Switching," Journal of the American Chemical Society, Vol. 126, No. 5, 2004, pp. 1493-1496. doi:10.1021/ja037118a

[15] J. Kim, S. Nayak and L. A. Lyon, "Bioresponsive Hydrogel Microlenses," Journal of the American Chemical Society, Vol. 127, No. 26, 2005, pp. 9588-9592. doi:10.1021/ja0519076

[16] Z. Hu and G. A. Huang, "A New Route to Crystalline Hydrogels, Guided by a Phase Diagram," Angewandte Chemie International Edition, Vol. 42, No. 39, 2003, pp. 4799-4802. doi:10.1002/anie.200351326

[17] H. Kawaguchi, K. Fujimoto and Y. Mizuhara, "Hydrogel microspheres III. Temperature-Dependent Adsorption of proteins on Poly-N-isopropylacrylamide Hydrogel Microspheres," Colloid and Polymer Science, Vol. 270, No. 1, 1992, pp. 53-57. doi:10.1007/BF00656929

[18] T. Sakai, Y. Hara and R. Yoshida, "Phase Transition Behaviors of Self-Oscillating Polymer and Nano-Gel Particles," Macromolecular Rapid Communications, Vol. 26, No. 14, 2005, pp. 1140-1144. doi:10.1002/marc. 200500195

[19] J. Shen, S. Pullela, M. Marquez and Z. Cheng, "Ternary Phase Diagram for the Belousov-Zhabotinsky ReactionInduced Mechanical Oscillation of Intelligent PNIPAM Colloids," Journal of Physical Chemistry A, Vol. 111, No. 48, 2007, pp. 12081-12085. doi:10.1021/jp072574x

[20] E. S. Matsuo and T. Tanaka, "Kinetics of Discon-Tinuous Volume-Phase Transition of Gels," Journal of Chemical Physics, Vol. 89, No. 3, 1988, pp. 1695-1703. doi: $10.1063 / 1.455115$

[21] T. Tanaka and D. J. Fillmore, "Kinetics of Swelling of Gels," Journal of Chemical Physics, Vol. 70, No. 3, 1979, pp. 1214-1218. doi:10.1063/1.437602

[22] E. C. Cho, J. W. Kim, A. Fernandez-Nieves and D. A. Weitz, "Highly Responsive Hydrogel Scaffolds Formed by Three-Dimensional Organization of Microgel Nanoparticles," Nano Letters, Vol. 8, No. 1, 2008, pp. 168172. doi:10.1021/n1072346e

[23] S. Maeda, Y. Hara, R. Yoshida and S. Hashimoto, "Peristaltic Motion of Polymer Gels," Angewandte Che- mie International Edition, Vol. 47, No. 35, 2008, pp. 66906693. doi:10.1002/anie. 200801347

[24] A. K. Dutt and M. Menzinger, "Stirring and Mixing Effects on Chemical Instabilities: Bistability of the Bromate/Bromide/Cerium(3+) System," Journal of Physical Chemistry, Vol. 94, No. 12, 1990, pp. 4867-4870. doi:10.1021/j100375a022 